\title{
A universal form of internal displacement field based quadrilateral area coordinate method QACM-II
}

\author{
Chen Xiaoming ${ }^{1, a}$, Duan Jin ${ }^{1}$ and Li Yungui ${ }^{1}$ \\ ${ }^{1}$ China state construction technical center, Beijing, China \\ aHanee@126.com
}

\begin{abstract}
Keywords: Quadrilateral area coordinate; Generalized conforming; Internal displacement field Abstract. Additional displacement fields based on internal parameters were used widely in finite element method to increase the order and complementary of displacement polynomials, thus accuracy and insensitivity to mesh distortion of elements can be improved significantly. To ensure that the element could pass patch test, internal displacement field should be formulated without incompatible energy on element's sides. In this paper, based on generalized conforming theory, the second quadrilateral area coordinate method QACM-II was used to develop universal form for plane elements. This universal internal displacement field can be used easily for those plane elements formulated with QACM-I or QACM-II.
\end{abstract}

\section{Introduction}

Based on Q4 element, professor Wilson[1] developed Q6 in 1973 by introducing additional displacement field formulated with internal parameters, so the displacement polynomials of Q6 element are quadratic complementary about isoparametric coordinate, and its accuracy and insensitivity to mesh distortion were improved significantly. As the additional displacement fields were only restrained by nodal conforming conditions, so Q6 element could not present exact solutions for patch test under irregular meshes.

Inspired by Q6 element, many 4-node non-conforming plane elements had been developed without any problem in convergence, such as the element QM6 proposed by Taylor et al. [2], QP6 by Wachspress [3], NQ6 by Pian et al. [4], the generalized conforming element GC-Q6 by Long et al. [5], the quasi-conforming element QC6 by Chen et al. [6], the hybrid-stress element P-S by Pian et al. [7], etc. All these elements can pass the strict form of patch test and possess excellent performance.

As most of these elements need additional displacement to improve the accuracy, then based on generalized conforming theory, a universal form of additional internal displacement field was developed by using isoparametric coordinate method[8]. This universal form can be used easily for improving the performance of quadrilateral plane elements.

In order to keep the elements insensitive under mesh distortion, Long et al. [9,10] developed the quadrilateral area coordinate QACM-I, Chen et al. [11] and Long et al. [12] developed the quadrilateral area coordinate QACM-II and QACM-III respectively.

In this paper, a universal form of internal displacement field was formulated by using QACM-II based on generalized conforming theory. This universal form can be used easily for those elements formulated with QACM-I or QACM-II, and it would improve the property without changing the compatibility of source elements.

\section{Introduction of QACM-II}

Compared with QACM-I, QACM-II contains only two independent coordinate components $\left(Z_{1}, Z_{2}\right)$. These two components were defined as :

$$
Z_{1}=4 \frac{A_{1}}{A}, \quad Z_{2}=4 \frac{A_{2}}{A}
$$

Where $A$ is the area of element, $A_{1}$ and $A_{2}$ are areas of $\triangle P M_{2} M_{4}$ and $\triangle P M_{3} M_{1}$ as shown in Fig.1. 


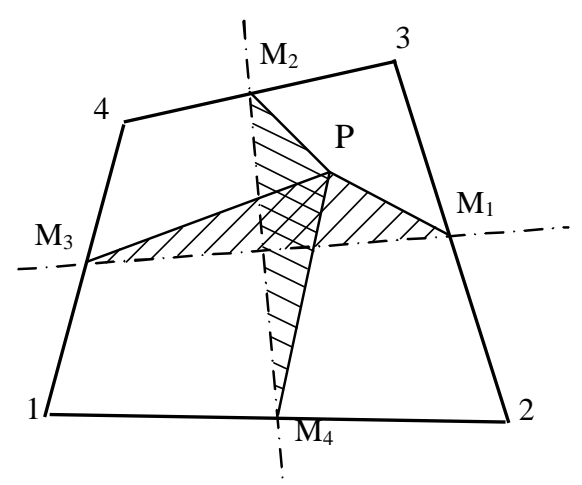

Fig.1 Definition of $Z_{1}$ and $Z_{2}$ of QACM-II

$$
\begin{aligned}
& A_{1}=S\left(\Delta P M_{2} M_{4}\right) \\
& A_{2}=S\left(\Delta P M_{3} M_{1}\right)
\end{aligned}
$$

$M_{i}(i=1,2,3,4)$ are the midpoints of elemental sides. Four shape parameters named $g_{i}(i=1,2,3,4)$ were defined in QACM-I as follows :

$$
\begin{array}{ll}
g_{1}=\frac{S(\Delta 124)}{A} & g_{2}=\frac{S(\Delta 123)}{A} \\
g_{3}=1-g_{1} & g_{4}=1-g_{2}
\end{array}
$$

Using these shape parameters, the relationship between QACM-II and Cartesian coordinates $(x, y)$ was defined as follows:

$$
\begin{aligned}
& Z_{1}=\frac{1}{A}\left[\bar{a}_{1}+\bar{b}_{1} x+\bar{c}_{1} y\right]+\left(g_{2}-g_{1}\right) \\
& Z_{2}=\frac{1}{A}\left[\bar{a}_{2}+\bar{b}_{2} x+\bar{c}_{2} y\right]+\left(g_{3}-g_{2}\right)
\end{aligned}
$$

Where

$$
\begin{aligned}
& a_{i}=x_{j} y_{k}-x_{k} y_{j}, \quad b_{i}=y_{j}-y_{k}, c_{i}=x_{k}-x_{j}, \quad i, j, k=1,2,3,4 \\
& \bar{a}_{1}=a_{3}-a_{1}, \bar{b}_{1}=b_{3}-b_{1}, \bar{c}_{1}=c_{3}-c_{1} \\
& \bar{a}_{2}=a_{4}-a_{2}, \bar{b}_{2}=b_{4}-b_{2}, \bar{c}_{2}=c_{4}-c_{2}
\end{aligned}
$$

And $x_{i}$ and $y_{i}$ are the coordinates of elemental nodes. The transformations of the derivatives of the first order can be written as: :

$$
\left\{\begin{array}{c}
\frac{\partial}{\partial x} \\
\frac{\partial}{\partial y}
\end{array}\right\}=\frac{1}{A}\left[\begin{array}{ll}
\bar{b}_{1} & \bar{b}_{2} \\
\overline{c_{1}} & \bar{c}_{2}
\end{array}\right]\left\{\begin{array}{c}
\frac{\partial}{\partial Z_{1}} \\
\frac{\partial}{\partial Z_{2}}
\end{array}\right\}
$$

The area integral formulae for the first, second terms are given by :

$$
\iint_{A}\left\{\begin{array}{l}
Z_{1} \\
Z_{2}
\end{array}\right\} d A=\frac{A}{3}\left\{\begin{array}{l}
g_{2}-g_{1} \\
g_{3}-g_{2}
\end{array}\right\}
$$




$$
\iint_{A}\left\{\begin{array}{c}
Z_{1}^{2} \\
Z_{2}^{2} \\
Z_{1} Z_{2}
\end{array}\right\} d A=\frac{A}{3}\left\{\begin{array}{c}
\left(g_{3}-g_{2}\right)^{2}+1 \\
\left(g_{2}-g_{1}\right)^{2}+1 \\
\left(g_{2}-g_{1}\right)\left(g_{3}-g_{2}\right)
\end{array}\right\}
$$

\section{Universal internal displacement field based on QACM-II}

In order to pass the patch test, the incompatible energy at element sides induced by internal displacement filed should be zero, then the conforming conditions that internal displacement field should be satisfied are as follows:

$$
\begin{aligned}
& \iint \frac{\partial \bar{u}}{\partial x} d A=f \mid l^{\prime} \bar{u} d s=0 \\
& \iint \frac{\partial \bar{u}}{\partial y} d A=f \mid m^{\prime} \bar{u} d s=0 \\
& \iint \bar{u} d A=0
\end{aligned}
$$

where $l^{\prime}$ and $m^{\prime}$ are the direction cosine of element sides.

As QACM-II has only two independent components $Z_{1}$ and $Z_{2}$, the universal displacement field based on internal parameters can be assumed as:

$$
\bar{u}=Z_{1}^{n} Z_{2}^{m}+\alpha_{1} Z_{1}+\alpha_{2} Z_{2}+\alpha_{0}
$$

By substituting Eq.10 into the former two equations of Eq.9, we have:

$$
\iint \frac{\partial \bar{u}}{\partial x} d A=\iint\left(\frac{\bar{b}_{1}}{A} \frac{\partial \bar{u}}{\partial Z_{1}}+\frac{\bar{b}_{2}}{A} \frac{\partial \bar{u}}{\partial Z_{2}}\right) d A=\frac{n \bar{b}_{1}}{A} \iint Z_{1}^{n-1} Z_{2}^{m} d A+\frac{m \bar{b}_{2}}{A} \iint Z_{1}^{n} Z_{2}^{m-1} d A+\bar{b}_{1} \alpha_{1}+\bar{b}_{2} \alpha_{2}=0
$$

and :

$$
\iint \frac{\partial \bar{u}}{\partial y} d A=\frac{n \bar{c}_{1}}{A} \iint Z_{1}^{n-1} Z_{2}^{m} d A+\frac{m \bar{c}_{2}}{A} \iint Z_{1}^{n} Z_{2}^{m-1} d A+\bar{c}_{1} \alpha_{1}+\bar{c}_{2} \alpha_{2}=0
$$

With Eq.11 and Eq.12, the undetermined parameters $\alpha_{1}$ and $\alpha_{2}$ can be derived as :

$$
\left\{\begin{array}{l}
\alpha_{1} \\
\alpha_{2}
\end{array}\right\}=-\frac{1}{A}\left[\begin{array}{ll}
\bar{b}_{1} & \bar{b}_{2} \\
\overline{c_{1}} & \bar{c}_{2}
\end{array}\right]^{-1}\left[\begin{array}{ll}
\bar{b}_{1} n & \bar{b}_{2} m \\
\overline{c_{1}} n & \bar{c}_{2} m
\end{array}\right]\left\{\begin{array}{l}
\iint Z_{1}^{n-1} Z_{2}^{m} d A \\
\iint Z_{1}^{n} Z_{2}^{m-1} d A
\end{array}\right\}=-\frac{1}{A}\left\{\begin{array}{l}
n \iint Z_{1}^{n-1} Z_{2}^{m} d A \\
m \iint Z_{1}^{n} Z_{2}^{m-1} d A
\end{array}\right\}
$$

By substituting Eq.10 into the third equation of Eq.9, we have:

$$
\iint \bar{u} d A=\iint Z_{1}^{n} Z_{2}^{m} d A-\frac{n}{A} \iint Z_{1}^{n-1} Z_{2}^{m} d A \iint Z_{1} d A-\frac{m}{A} \iint Z_{1}^{n} Z_{2}^{m-1} d A \iint Z_{2} d A+\alpha_{0} A=0
$$

Then the undetermined parameters $\alpha_{0}$ can be written as:

$$
\alpha_{0}=-\frac{1}{A} \iint Z_{1}^{n} Z_{2}^{m} d A+\frac{n}{A^{2}} \iint Z_{1}^{n-1} Z_{2}^{m} d A \iint Z_{1} d A+\frac{m}{A^{2}} \iint Z_{1}^{n} Z_{2}^{m-1} d A \iint Z_{2} d A
$$

The integration in Eq.15 can be denoted as:

$$
I_{n, m}^{Z}=\iint Z_{1}^{n} Z_{2}^{m} d A
$$

Then $\alpha_{0}$ and the additional displacement field can be written as follows:

$$
\begin{aligned}
& \alpha_{0}=-\frac{1}{A} I_{n, m}^{Z}+\frac{n}{A^{2}} I_{n-1, m}^{Z} I_{1,0}^{Z}+\frac{m}{A^{2}} I_{n, m-1}^{Z} I_{0,1}^{Z} \\
& \bar{u}=Z_{1}^{n} Z_{2}^{m}-\frac{n}{A} I_{n-1, m}^{Z} Z_{1}-\frac{m}{A} I_{n, m-1}^{Z} Z_{2}+\alpha_{0}
\end{aligned}
$$


Examples of quadratic term are presented as follows:

When $n=2, m=0$ :

$$
\begin{aligned}
& \bar{u}=Z_{1}^{2}+\alpha_{1} Z_{1}+\alpha_{0} \\
& \alpha_{1}=-\frac{2}{A} \iint Z_{1} d A=-\frac{2}{3}\left(g_{2}-g_{1}\right) \\
& \alpha_{0}=-\frac{1}{A} \iint Z_{1}^{2} d A+\frac{2}{A^{2}} \iint Z_{1} d A \iint Z_{1} d A=-\frac{1}{3}\left[\left(g_{3}-g_{2}\right)^{2}+1\right]+\frac{2}{9}\left(g_{2}-g_{1}\right)^{2}
\end{aligned}
$$

When $n=1, m=1$ :

$$
\begin{aligned}
& \bar{u}=Z_{1} Z_{2}+\alpha_{1} Z_{1}+\alpha_{2} Z_{2}+\alpha_{0} \\
& \alpha_{1}=-\frac{1}{A} \iint Z_{2} d A=-\frac{1}{3}\left(g_{3}-g_{2}\right) \\
& \alpha_{2}=-\frac{1}{A} \iint Z_{1} d A=-\frac{1}{3}\left(g_{2}-g_{1}\right) \\
& \alpha_{0}=-\frac{1}{A} \iint Z_{1} Z_{2} d A+\frac{2}{A^{2}} \iint Z_{1} d A \iint Z_{2} d A=-\frac{1}{9}\left(g_{2}-g_{1}\right)\left(g_{3}-g_{2}\right)
\end{aligned}
$$

When $n=0, m=2$ :

$$
\begin{aligned}
& \bar{u}=Z_{2}^{2}+\alpha_{2} Z_{2}+\alpha_{0} \\
& \alpha_{2}=-\frac{2}{A} \iint Z_{2} d A=-\frac{2}{3}\left(g_{3}-g_{2}\right) \\
& \alpha_{0}=-\frac{1}{A} \iint Z_{2}^{2} d A+\frac{2}{A^{2}} \iint Z_{2} d A \iint Z_{2} d A=-\frac{1}{3}\left[\left(g_{2}-g_{1}\right)^{2}+1\right]+\frac{2}{9}\left(g_{3}-g_{2}\right)^{2}
\end{aligned}
$$

Examples of cubic term are presented as follows:

When $n=3, m=0$

$$
\begin{aligned}
& \bar{u}=Z_{1}^{3}+\alpha_{1} Z_{1}+\alpha_{0} \\
& \alpha_{1}=-\frac{3}{A} \iint Z_{1}^{2} d A=-\left[\left(g_{3}-g_{2}\right)^{2}+1\right] \\
& \alpha_{0}=-\frac{1}{A} \iint Z_{1}^{3} d A+\frac{3}{A^{2}} \iint Z_{1}^{2} d A \iint Z_{1} d A=\frac{2}{15}\left(g_{2}-g_{1}\right)\left[\left(g_{3}-g_{2}\right)^{2}+1\right]
\end{aligned}
$$

When $n=2, m=1$

$$
\begin{aligned}
\bar{u} & =Z_{1}^{2} Z_{2}+\alpha_{1} Z_{1}+\alpha_{2} Z_{2}+\alpha_{0} \\
\alpha_{1} & =-\frac{2}{A} \iint Z_{1} Z_{2} d A=-\frac{2}{3}\left(g_{2}-g_{1}\right)\left(g_{3}-g_{2}\right) \\
\alpha_{2} & =-\frac{1}{A} \iint Z_{1}^{2} d A=-\frac{1}{3}\left[\left(g_{3}-g_{2}\right)^{2}+1\right] \\
\alpha_{0} & =-\frac{1}{A} \iint Z_{1}^{2} Z_{2} d A+\frac{2}{A^{2}} \iint Z_{1} Z_{2} d A \iint Z_{1} d A+\frac{1}{A^{2}} \iint Z_{1}^{2} d A \iint Z_{2} d A \\
& =-\frac{1}{15}\left\{\left(g_{3}-g_{2}\right)^{3}+2\left(g_{3}-g_{2}\right)\left(g_{2}-g_{1}\right)^{2}+5\left(g_{3}-g_{2}\right)\right\} \\
& +\frac{2}{9}\left(g_{3}-g_{2}\right)\left(g_{2}-g_{1}\right)^{2}+\frac{1}{9}\left(g_{3}-g_{2}\right)\left[\left(g_{3}-g_{2}\right)^{2}+1\right]
\end{aligned}
$$

When $n=1, m=2$ 


$$
\begin{aligned}
\bar{u} & =Z_{1} Z_{2}^{2}+\alpha_{1} Z_{1}+\alpha_{2} Z_{2}+\alpha_{0} \\
\alpha_{1} & =-\frac{1}{A} \iint Z_{2}^{2} d A=-\frac{1}{3}\left[\left(g_{2}-g_{1}\right)^{2}+1\right] \\
\alpha_{2} & =-\frac{2}{A} \iint Z_{1} Z_{2} d A=-\frac{2}{3}\left(g_{2}-g_{1}\right)\left(g_{3}-g_{2}\right) \\
\alpha_{0} & =-\frac{1}{A} \iint Z_{1} Z_{2}^{2} d A+\frac{1}{A^{2}} \iint Z_{2}^{2} d A \iint Z_{1} d A+\frac{2}{A^{2}} \iint Z_{1} Z_{2} d A \iint Z_{2} d A \\
& =-\frac{1}{15}\left\{\left(g_{2}-g_{1}\right)^{3}+2\left(g_{3}-g_{2}\right)^{2}\left(g_{2}-g_{1}\right)+5\left(g_{2}-g_{1}\right)\right\} \\
& +\frac{1}{9}\left(g_{2}-g_{1}\right)\left[\left(g_{2}-g_{1}\right)^{2}+1\right]+\frac{2}{9}\left(g_{2}-g_{1}\right)\left(g_{3}-g_{2}\right)^{2}
\end{aligned}
$$

When $n=0, m=3$

$$
\begin{aligned}
& \bar{u}=Z_{2}^{3}+\alpha_{2} Z_{2}+\alpha_{0} \\
& \alpha_{2}=-\frac{3}{A} \iint Z_{2}^{2} d A=-\left[\left(g_{2}-g_{1}\right)^{2}+1\right] \\
& \alpha_{0}=-\frac{1}{A} \iint Z_{2}^{3} d A+\frac{3}{A^{2}} \iint Z_{2}^{2} d A \iint Z_{2} d A=\frac{2}{15}\left(g_{3}-g_{2}\right)\left[\left(g_{2}-g_{1}\right)^{2}+1\right]
\end{aligned}
$$

This universal form can be used directly as the shape function of additional displacement based on internal parameters for those plane elements formulated with QACM-I or QACM-II. If the nodal displacement was compatible, the new element would pass the strict form of patch test.

\section{Conclusions}

Additional displacement field based on internal parameters are important for developing 4-node plane elements. In this paper, based on generalized conforming conditions, an universal form of internal displacement has been formulated with quadrilateral area coordinate QACM-II, and the quadratic and cubic polynomials are also presented as examples for other researchers. These universal polynomials can be used easily for those plane elements developed with QACM-I or QACM-II.

\section{References}

[1] E L Wilson, R L Taylor, et al., Incompatible displacement models, Numerical and Computer Methods in Structural Mechanics, In: S J Fenves et al., eds, Academic Press, New York, 1973, 43-57. [2] R. L. Taylor, P. J. Beresford, E. L. Wilson. A non-conforming element for stress analysis. International Journal for Numerical Methods in Engineering, Vol. 10, pp. 1211-1219, 1976.

[3] E.L. Wachspress. Incompatible quadrilateral basis functions. International Journal for Numerical Methods in Engineering, Vol. 12, pp. 589-595, 1978.

[4] T.H.H. Pian, C. C. Wu. Hybrid and Incompatible Finite Element Methods. Chapman \& Hall/CRC, Boca Raton, 2006.

[5] Y. Q. Long, M. F. Huang. A generalized conforming isoparametric element. Applied Mathematics and Mechanics-English Edition, Vol. 10, pp. 929-936, 1988.

[6] W. J. Chen, L. M. Tang. Isoparametric quasi-conforming element. Journal of Dalian University of Technology, Vol. 20, no. 1, pp. 63-74, 1981.

[7] T. H. H. Pian, K. Sumihara. Rational approach for assumed stress finite elements. International Journal for Numerical Methods in Engineering, Vol. 20, pp. 1685-1695, 1984.

[8] Zhang Chunsheng, Long Yuqiu, Xu Yin. Three-dimensional basic formulations of additional incompatible displacement of internal parameters. Engineering Mechanics. 2011, 18(5): 50-63.

[9] N S Lee, K J Bathe. Effects of element distortion on the performance of isoparametric elements. Int. J. Num. Meth. Engng. 1993(36): 3553-3576. 
[10] Y.Q. Long, J.X. Li, Z.F. Long, and S. Cen. Area coordinates used in quadrilateral elements. Communications in Numerical Methods in Engineering, vol. 15, no. 8, pp. 533-545, 1999.

[11]Z.F. Long, J.X. Li, S. Cen, et al. Some basic formulae for Area coordinates used in quadrilateral elements. Communications in Numerical Methods in Engineering, vol. 15, no.12, pp. 841-852, 1999. [12]X.M. Chen, S. Cen, X.R. Fu, and Y.Q. Long, "A new quadrilateral area coordinate method (QACM-II) for developing quadrilateral finite element models," International Journal for Numerical Methods in Engineering, vol. 73, no. 13, pp. 1911-1941, 2008.

[13]Z.F. Long, S. Cen, L. Wang, X.R. Fu, and Y.Q. Long, "The third form of the quadrilateral area coordinate method (QACM-III): Theory, application, and scheme of composite coordinate interpolation," Finite Elements in Analysis and Design, vol. 46, no. 10, pp. 805-818, 2010. 